\title{
Eine integrierte betriebswirtschaftliche Fallstudie mit SAP ERP und deren Einbindung in das SAP LEGO Labor
}

\author{
Danny Szendrei, Tobias Teich, Katja Unger, Jörg Militzer \\ Fachbereich Wirtschaftswissenschaften, \\ Westsächsische Hochschule Zwickau
}

\section{Einleitung}

Der folgende Beitrag stellt ein mehrgliedriges Pädagogikkonzept zur Integration komplexer betriebswirtschaftlicher Inhalte in Form einer ERP-Fallstudie vor.

Mit dem ERP-System als Lernträger werden modul- bzw. studiengangspezifische Inhalte aller Studienangebote der Fakultät Wirtschaftswissenschaften auf einer Plattform integriert. Das Fördern von abteilungsübergreifendem Verständnis der interdisziplinären Zusammenhänge strategischer und taktischer Unternehmensziele sowie produktionswirtschaftlicher Inhalte soll mittels der integrierten Fallstudie lebensnah angeboten werden. Durch die Einbindung der Fallstudie in ein Laborumfeld können insbesondere Logistikprozesse visualisiert und somit aktiv von Studierenden mitgestaltet werden. Mit der Fallstudie wird einerseits eine moderne, prozessorientierte Lehr- und Ausbildungsplattform geschaffen. Andererseits integriert die Fachgruppe Wirtschaftsinformatik die Bedeutung von aktuell geforderten Kompetenzprofilen seitens der Industrie in die akademische Lehre.

\section{Motivation}

Integrierte Informationssysteme bilden einen entscheidenden, zukunftsweisenden und beliebten Bestandteil akademischer Forschung und Lehre (Unger et al. 2008, S. 97). An der Westsächsischen Hochschule Zwickau sind ERP-Systeme seit 2004 wichtiger Bestandteil der akademischen Ausbildung. Die integrierte Fallstudie mit

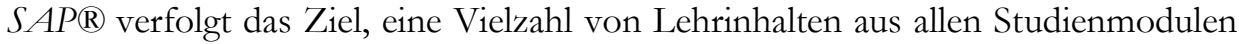
der Fakultät Wirtschaftswissenschaften als kompakte Fallstudie in einem ERPSystem zu verdichten. Die große Nachfrage seitens der Studierenden sowie die positiven Bewertungen des Kurses (Bewertung aus MeinProf.de, 2009) ermöglichen ein personelles und inhaltliches Weiterentwickeln der Kursinhalte. Das Kon- 
zept des Kurses stieß während der Hannover Messe 2009 auf großes Interesse und wurde positiv bewertet. ${ }^{1}$

Die Fachgruppe Wirtschaftsinformatik reagiert mit diesem Kursangebot auf die große Nachfrage seitens der Industrie, eine breite, qualitativ hochwertige Ausbildung in ERP-Systemen für Studierende anzubieten. Neben der Eigenschaft als Lehrplattform für Studierende kommt der Fallstudie noch eine weitere Bedeutung für Forschung und Entwicklung zu. Diese Funktion ist an interessierte, engagierte Studenten gerichtet, die durch ihre Arbeit die Kursinhalte erweitern und aktualisieren. Gleichsam bildet die Fallstudie in Verbindung mit dem SAP LEGO Labor für diese Studierenden eine Plattform für Analyse und Visualisierung aktueller Strategien und Geschäftsprozesse aus dem Bereich Unternehmenslogistik.

\section{Abgrenzung zu anderen Konzepten}

Die große Bedeutung, die der Vermittlung praxisrelevanten Wissens bei gleichzeitiger Förderung von Methoden- und sozialen Kompetenzen zukommt, scheint unbestritten. Die Fallstudie setzt deshalb auf eine Vielfalt von Geschäftsvorfällen aus allen Teilbereichen eines Produktionsbetriebes und die Kommunikation der Teilnehmer am SAP-System sowie persönliche Kommunikation. Die Geschäftsvorfälle werden transaktionsbasiert an der Benutzeroberfläche von SAP ERP / SCM bearbeitet. Als wichtiges Element dieser Fallstudie ist die Vor- und Nachbereitung der Geschäftsprozesse in Form von interaktiven Lehrvorträgen bzw. begleitenden (Rechen-) Aufgaben zu beschreiben. Somit grenzt sich die Fallstudie von klassischen Präsenz- und Frontallehrangeboten ab (Eicker und Mense 2007, S. 59). Die Integration von Planungs-, Durchführungs- als auch Bewertungs- / Kontrollprozessen - im Zusammenhang mit der Kursaufbereitung durch studentische Dozenten - kann konzeptuell durchaus als multiperspektivisch beschrieben werden. Dabei grenzt sich diese Auffassung von Multiperspektive der Sichtweise Dauns et.al. (2006, S. 4) ab. Dort werden Konzepte und Softwarestrukturen bestimmter ERP-Systeme in deren Vertiefung unter diesem Begriff subsummiert. Eine Ausrichtung der Fallstudie auf die Vermittlung von IT-Fachinhalten (z. B. Ebenenarchitektur, ABAP-Programmierung) wird vermieden. Eine weitere Abgrenzung erfolgt in der Gestaltung der Inhalte, die durch Studierende erfolgt. Diese bereiten fachspezifisches Wissen auf und fungieren in nachfolgenden Kursen als Dozenten. Damit wird eine tiefere Ausprägung sozialer Kompetenzen (z. B. Teamfähigkeit, Konfliktlösung, Kommunikationsbereitschaft) angestrebt, wie sie nach Ansicht der Autoren in reinen E-Learning oder Frontalangeboten nicht erreicht werden kann.

${ }^{1}$ http://www.heise.de/newsticker/Hannover-Messe-SAP-Ausbildung-mit-Lego--/meldung/136598 


\section{$4 \quad$ Inhalte und Zielsetzung}

\subsection{Inhalte der Fallstudie}

Die Fallstudie wird an der Westsächsischen Hochschule Zwickau in der Fachgruppe Wirtschaftsinformatik angeboten. In den traditionellen Diplomstudiengängen BWL, Wirtschaftsingenieurwesen sowie Management öffentlicher Aufgaben konnte sie als Wahlpflichtfach besucht werden. Mit Einführung der BachelorStudiengänge für BWL und Management öffentlicher Aufgaben wurde sie als Pflichtbestandteil in das Modul ERP-Systeme aufgenommen.

Kerninhalte der Fallstudie bilden Planung, Strukturierung, Aufbau und operativer Betrieb einer beispielhaften Supply Chain (SC) zur Stuhlproduktion. Diese besteht aus drei Unternehmen unterschiedlicher Wertschöpfungstiefe ( $1^{\text {st }}$ Tier und $2^{\text {nd }}$ Tier- Supplier, Finalproduzent), die gegenseitig in Zuliefer- und Abnehmerbeziehungen stehen. Die SC besteht aus den Unternehmen Plaste AG, Polster GmbH sowie Stuhlwerke Zwickau GmbH. Dabei liefert die Plaste AG Rohformteile aus Plastik an die Poster GmbH und Fertigerzeugnisse an die Stuhlwerke. Die Polster GmbH veredelt die Rohformteile zu Fertigerzeugnissen (gepolsterte Lehnund Sitzteile) und liefert diese wiederum an die Stuhlwerke.

Dieses Unternehmen fertigt Stuhlgestelle und montiert diese dann mit den Zuliefermaterialien zu einem absatzfertigen Bürostuhl.

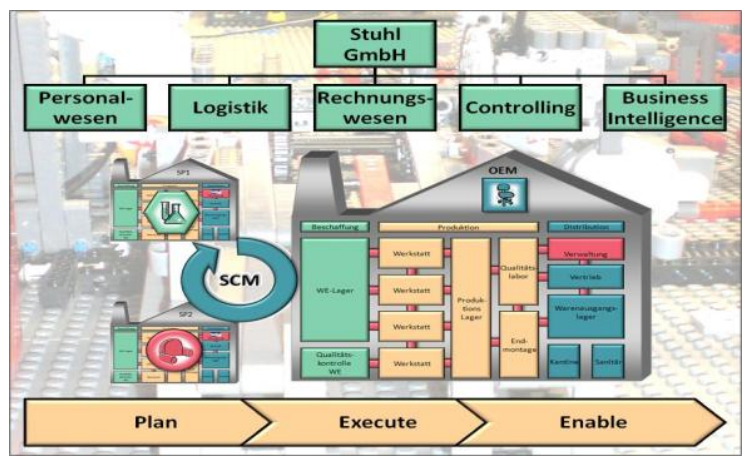

Abbildung 1: Schematische Darstellung der Supply Chain Struktur

Um diese Wirtschaftsbeziehungen abbilden und bedienen zu können, werden während des Kursverlaufs sämtliche Unternehmensressourcen und Informationsbeziehungen in das ERP-System eingepflegt. Als besonderes Merkmal ist hervorzuheben, dass alle Unternehmen durch die Teilnehmer ,auf der grünen Wiese“, also ohne jegliche systemseitige Voreinstellungen, aufzubauen sind. Zum weiteren Verständnis der organisatorischen, physischen sowie betriebswirtschaftlichen Zusammenhänge dieser SC werden die Unternehmen im SAP LEGO Labor als physische Modelle abgebildet und relevante Geschäftsprozesse auf dieser Plattform funktio- 
nal umgesetzt. Dabei erfolgt die Ansteuerung der einzelnen Funktionselemente wie Transportressourcen oder Handlingeinheiten aus dem SAP ERP-System in Anlehnung an vorher erfolgte, systemseitige Buchung von Geschäftsvorfällen. Weiterführend werden die Labormodelle samt ihrer Parameter für eine visuelle Darstellung in der Software vis'TABLE abgebildet.

\subsection{Zielsetzung}

Mit der Fallstudie, sowie deren Einbindung in eine Laborlandschaft, wird das Ziel verfolgt, die vielfältigen Inhalte der akademischen, betriebswirtschaftlichen Ausbildung integrativ, praxisrelevant und eigenverantwortlich anzubieten.

Im Vordergrund steht das Fördern von abteilungs- bzw. studiengangsübergreifendem Lernen und Anwenden angebotener Lehrinhalte aus diversen Studienmodulen. Zu diesem Zweck wurden aus den an der Fakultät Wirtschaftswissenschaften angebotenen Lehrinhalten aktuelle, praxisrelevante Inhalte zu beispielhaften, dabei fachlich untersetzten Geschäftsvorfällen aus dem Unternehmensalltag aggregiert. Das Fördern von methodischen und fachlichen Kompetenzen zur Planung und Organisation komplexer Inhalte wird in der Fallstudie mit der Einführung zukunftsweisender Technologien, z. B. RFID, angereichert.

Den Studierenden wird nach Teilnahme des Kurses angeboten, sich selbst in das Team einzubringen um dort individuelle, studienschwerpunktbegleitende Interessen in den einzelnen Kernfeldern der Fallstudie zu vertiefen. Selbstverständlich werden dabei durch die Studierenden immer wieder neue Kursinhalte eingebracht, die weitgehend eigenverantwortlich ausgearbeitet werden. Die Inhalte werden jeweils zum nächsten Kurs von den neuen Teammitgliedern vorgetragen und betreut.

In den vergangenen Jahren konnte das große Interesse der Studierenden genutzt werden, um immer neue Dozenten mit der Erarbeitung und Aufbereitung neuer und aktueller Geschäftsvorfälle zu gewinnen. Dabei setzen sich Studierende und Dozenten mit den Herausforderungen einer versatilen, komplexen Informationslandschaft in den Unternehmen auseinander. Im Vordergrund stehen hierbei die Bewältigung von Schnittstellenproblematiken der Informationen aus den einzelnen betriebswirtschaftlichen Kernfeldern und deren Zusammenfassung zu durchführbaren, integrativen Fallstudieninhalten. Als Fallstudieninhalte sind derzeit die Umsetzung von pull-Prozessen, lean management-Ansätzen sowie der Auftragsoptimierung nach Reihenfolgerestriktionen in der SAP Business Suite in Planung. Diese Inhalte bieten sich durch die Möglichkeit zur Visualisierung im Labor sowie aktueller Nachfragen von Industriepartnern der Hochschule an. 


\section{Betriebswirtschaftliche Kerninhalte der Supply Chain}

Wie bereits dargestellt, stehen in der Fallstudie Unternehmen in gegenseitigen Liefer- und Abnehmerbeziehungen. Um die Szenarien aus Material-, Informationsund Finanzflüssen der Unternehmen vielfältig und praxisrelevant darzustellen, werden in der Fallstudie Geschäftprozesse innerhalb der jeweiligen Kernfelder aufbereitet. Die Kernfelder umfassen die Bereiche:

Customizing, Personal, Logistik, Rechnungswesen, Contolling und Business Intelligence mit den Anwendungen aus folgendem Schema:

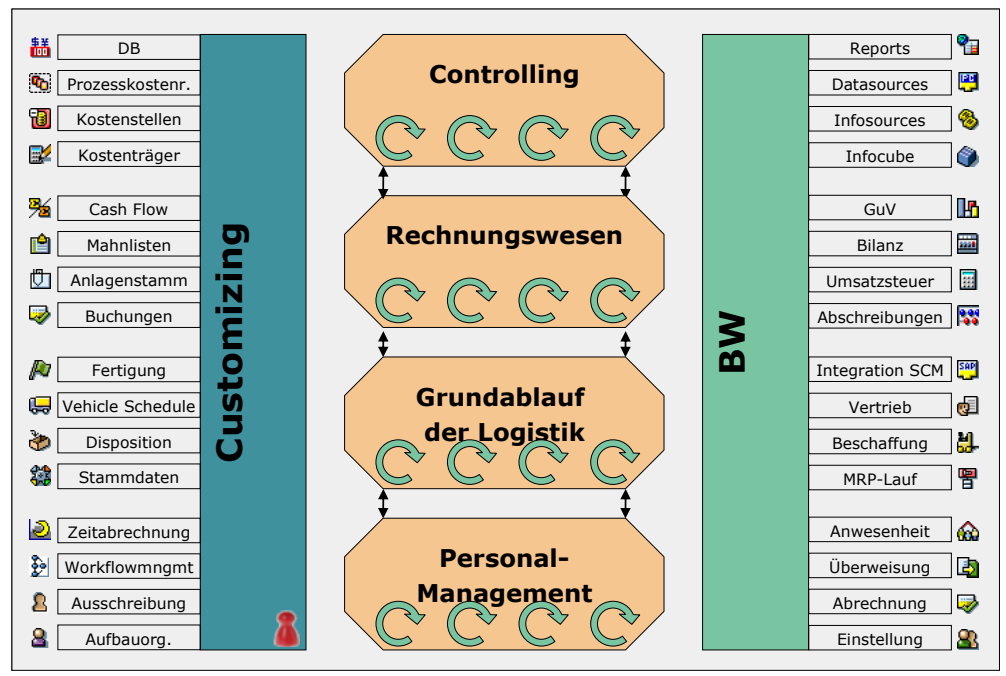

Abbildung 2: Ablaufstruktur der betriebswirtschaftlichen Kernfelder

Ausnahme bildet hierbei das Customizing. Dieses bildet keine betriebswirtschaftliche Kernaufgabe im klassischen Sinne, wird jedoch im Hinblick auf die Durchführbarkeit von Geschäftsprozessen in den Kernfeldern systemseitig bedingt. Somit kommt dem Customizing zwar eine periphere, jedoch zentrale Bedeutung zu.

\subsection{Customizing}

In diesem Kernfeld werden die Organisationsstrukturen und Verknüpfungen von Unternehmen und deren Prozessen für die Fallstudie in SAP ERP bzw. SAP SCM im SAP System angelegt. Dem Customizing kommen im Umfeld der zweiten Fallstudie zentrale Bedeutungen zu. Einerseits wird den Teilnehmern die Bedeutung der Adaption des ERP-Systems an die bestehenden Strukturen der SC vermittelt. Weiterführend kann in Vorbereitung oder Aktualisierung von Geschäftsprozessen für die Fallstudie aktuelle wissenschaftliche Diskussion über process alignment versus ERP customization nachvollzogen werden (Grabot et.al. 2008, S.139). In der Ausarbeitung neuer Inhalte der Fallstudie, ungeachtet des jeweiligen Kernfel- 
des, stellt sich das Customizing in SAP ERP als Plattform für Change Management dar. Die Herausforderung der Dozenten ist also die strategische Planung und Implementierung des Geschäftsprozesses in Wechselwirkung mit bestehenden Inhalten.

\subsection{Personal}

In Kernfeld Personal werden organisatorische Bedingungen sowie Geschäftsprozesse aus dem Bereich Human Resources dargestellt und umgesetzt. Begleitet wird auch dieses Feld von diversen Einstellungen im Customizing.

Inhalte dieses Kernfeldes bilden Prozesse zu Bewerbung, Bewerberauswahl und -einstellung. Weiterführend werden Gehaltsbuchungen und Arbeitszeitmodelle bearbeitet.

Über die Beantragung und Genehmigung von Urlaubstagen wird ein kompakter Workflow durch die Teilnehmer erstellt.

Im Jahr 2008 wurde erstmalig eine Zeiterfassung und Verbuchung über Terminals durch die Dozenten in die Fallstudie implementiert. So können die Kursteilnehmer selbst aktiv den Zeiterfassungsprozess durchführen und verbuchen.

\subsection{Logistik}

Im Kernfeld Logistik werden nach Einpflegen von Stammdaten (Materialstämme, Stücklisten etc.) alle Planungsaufgaben im Vorfeld der physischen Wertschöpfung vorgenommen. Über die Programmplanung (Produktionsprogramm, Ablaufplanung) erfolgt die Einplanung der Fertigungsaufträge anhand verschiedener Rahmenbedingungen. Weiterhin werden Analysen und Prognoseverfahren vorgestellt und ausgeführt. Nach einer systemgestützten Lieferantenbewertung und deren Auswahl werden verschiedene Konzepte des Beschaffungsprozesses im SAPSystem (Bestellanforderung - Bestellung - Transportauftrag vs. Umlagerungsbestellung) vorgestellt.

Die integrative Transportplanung der zu distribuierenden Fertigerzeugnisse ist Bestandteil des Moduls SAP SCM.

Weiterführend werden derzeit Konzepte entwickelt, die Kapazitätsplanung und Auftragsterminierung zwischen den Unternehmen ebenfalls über SAP SCM auszuführen.

\subsection{Rechnungswesen}

Inhalt dieses Kernfeldes ist die Bewertung und Verbuchung sämtlicher ausgeführten Transaktionen der Fallstudie. Voraussetzungen hierfür sind die Pflege von entsprechenden Nummernkreisen für die Organisationsstrukturen im Customizing. Ferner werden in diesem Kernfeld Vermögenspositionen im Aktiva erstellt und bebucht. Die Buchhaltung wird mittels Bilanzierung sowie Gewinn- und Ver- 
lustrechnung durchgeführt. Ferner wird eine Cash Flow Analyse im eigenen Buchungskreis vorgenommen.

\subsection{Controlling}

Um den Studierenden ein reales Szenario, das sich von akademischen Rechenbeispielen des internen Rechnungswesens abhebt, zu präsentieren, werden in der Fallstudie verschiedene Kalkulationsverfahren mit SAP ERP durchgeführt. Über die Produktkostenkalkulation der jeweils eigenen Fertigerzeugnisse erfolgen Kostenstellenrechnungen und aktuell auch Prozesskostenrechnungen. Abgerundet wird das Controlling durch die schrittweise Erarbeitung einer Formelberechnung für die Deckungsbeitragsrechnung.

\subsection{Business Intelligence (BI)}

In den bislang beschriebenen Kernfeldern der Fallstudie wurden unternehmensspezifische Stammdaten gepflegt. Diese wurden dann in der Ausführung von Geschäftsprozessen durch Bewegungsdaten untersetzt, die nunmehr im System vorliegen. Inhalt des Kernfeldes BI ist die erfolgsrelevante Auswertung der vorliegenden Informationen. Dazu werden durch die Studierenden mehrdimensionale Datenwürfel (InfoCubes) angelegt, die schrittweise mit InfoObjects (Merkmalen und Kennzahlen) angereichert und verdichtet werden. Abschließend werden exemplarische Anfragen (Queries) an das BW-System erarbeitet, die zur Anzeige erfolgswirksamer, strategischer Kennzahlen führen.

\section{Pädagogikkonzept der Fallstudie}

Die integrierte Fallstudie wird pro Semester einmalig angeboten. Der Kurs dauert zwei Wochen und ist als Präsenzveranstaltung über volle Tage konzipiert. Voraussetzung zur Kursanmeldung bildet die Teilnahme an der Vorlesungsreihe ERPSysteme für die Studierenden der Diplomstudiengänge.

Zur Einführung in den Kurs werden das Produkt SAP Business Suite sowie die Wirtschaftsbeziehungen der drei Beispielunternehmen vorgestellt. Im weiteren Verlauf sind sämtliche Inhalte den bereits beschriebenen Kernfeldern zugeordnet.

Die Bearbeitung der Inhalte erfolgt während des Kursverlaufs anhand von drei pädagogischen Konzepten:

\subsection{Interaktive Lerneinheiten}

Dieses Konzept beinhaltet die Vorstellung der akademischen, betriebswirtschaftlichen Inhalte. Darin werden Lehrinhalte, z. B. Planungsalgorithmen, von den Dozenten erläutert und gemeinsam mit den Teilnehmern erörtert. Die Einbeziehung 
der Teilnehmer verfolgt das Ziel, bisheriges Vorwissen der Studierenden zu erfragen und neue Informationen weiterführend anzubieten. Die Lerneinheiten stehen als PDF-Dokument mit dem Verweis zur jeweils anschließenden Trainingseinheit am ERP-System zum Download zur Verfügung. Diese Lerneinheiten sind den Trainingseinheiten vorangestellt und dienen zur Einführung in den jeweiligen Ausbildungsabschnitt.

Die Dozenten rekrutieren sich ausschließlich aus Studierenden, die die Fallstudie bereits in vergangenen Semestern besuchten. Die Aufbereitung der Lehrinhalte sowie die rhetorische Umsetzung der Inhalte erfolgt eigenverantwortlich, wird aber selbstverständlich vom Teamwork der Dozenten maßgeblich verbessert. Da spezifische Inhalte eines Kernfeldes oftmals auch Auswirkungen auf andere Bereiche haben, z. B. das Verbuchen von Beständen, wird pro Kernfeld ein Bereichsleiter bestimmt. Dieser koordiniert sowohl Aufgaben als auch deren schriftliche Einarbeitung in die Kursunterlagen.

\subsection{Begleitaufgaben}

Während des Kursverlaufes werden eine Vielzahl von Geschäftsprozessen im ERP-System bearbeitet. Vielen Prozessen geht eine Planung der durchzuführenden Aufgabenstellung (z. B. Materialbedarfsplanung, Prognoserechnung) voraus. Um den Studierenden die Komplexität der Planungsaufgaben, und somit die enorme Leistungsfähigkeit von SAP ERP zu verdeutlichen, werden Begleitaufgaben mit den Inhalten der Fallstudie bereitgestellt. Ziel dieser Aufgaben ist die Auseinandersetzung mit den betriebswirtschaftlichen Größen im Rahmen einer manuellen Beplanung der Aufgaben. Dazu sind die entsprechenden Informationen und Rahmenbedingungen aus der Fallstudie in diverse Planungstabellen zu übernehmen. Die Teilnehmer führen dort eigene Prognosen bzw. Planungen durch, die später mit den Ergebnissen aus dem ERP-System verglichen werden. Die Bearbeitung der Begleitaufgaben dient ebenfalls der Überbrückung zeitlicher Zwangspausen, die durch Customizing-Einstellungen verursacht werden. Der Lösungsweg wird von den Dozenten des jeweiligen Kernfeldes begleitet.

\subsection{Angeleitetes Selbststudium}

Im Rahmen dieses Pädagogikmoduls erarbeiten sich die Studierenden die Inhalte und Benutzereingaben in SAP ERP eigenverantwortlich anhand verfügbarer Kursdokumente. Dazu zählen die als PDF bereitgestellten Trainingseinheiten, als auch das eigens dafür herausgegebene Lehrbuch Eine integrierte betriebswirtschaftliche Fallstudie mit SAP® (Käschel und Teich 2008). Die Inhalte der Lehrmittel unterliegen einem ständigen Wandel. Neue bzw. modifizierte Inhalte der Fallstudie werden in die Folgeauflagen des Lehrbuches übernommen.

Darin finden die Teilnehmer die zu bearbeitenden Customizing-Einstellungen und Geschäftsprozesse der entsprechenden Trainingseinheit. Ein Gleichstand des 
Arbeitsfortschrittes der Kursteilnehmer wird durch die Lerneinheiten vor jeder Trainingseinheit erreicht. Durch die Untersetzung der Geschäftsprozesse mit EPK's (Ereignis-gesteuerte Prozessketten) wird das Prozessverständnis in den einzelnen Kernfeldern gefördert.

\section{Integration neuer Technologien und weiterführende Konzepte}

Ergänzt werden die gezeigten Pädagogikkonzepte durch die Visualisierung der Geschäftsprozesse im SAP LEGO Labor. Darin sind, wie bereits beschrieben, Modelle der Unternehmen angelegt.

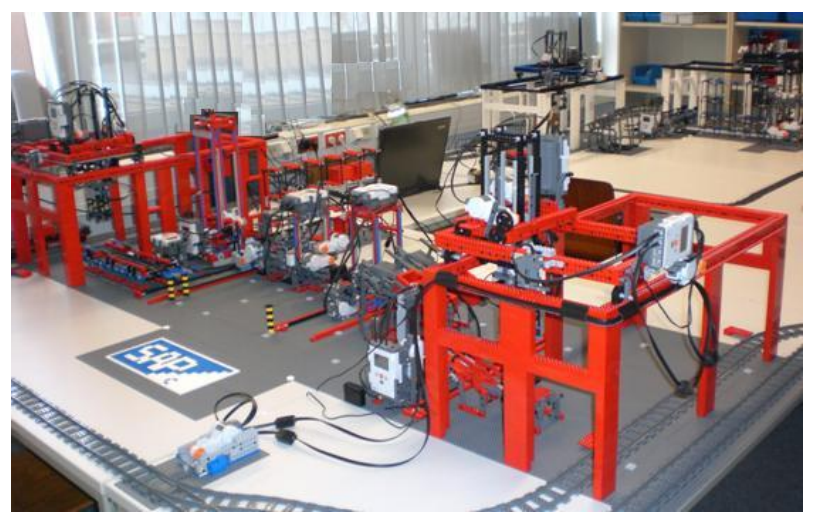

\section{Abbildung 3: LEGO-Modell OEM}

Die Verwendung programmierbarer Speicherbausteine aus der Produktreihe NXT Mindstorms der Firma LEGO erlaubt sowohl die physische Darstellung von Liefer- oder Lagerprozessen als auch die Programmierung lokaler Geschäftsprozesse. Als Schnittstelle zwischen LEGO-Landschaft und dem ERP-System dient ein Steuerrechner, der Informationen aus dem ERP ausliest und entsprechend hinterlegte Laborabläufe ansteuert. Exemplarisch werden für die Fallstudie Bestellabrufe und Fertigungsaufträge vorgenommen, die die Teilnehmer selbst im ERP pflegen.

Die Verpackungseinheiten der Unternehmen wurden in SAP ERP als handling units angelegt und sind mit RFID Technik bestückt. Damit wird ein lückenloses Tracking der units und des Materials im Transport und Warehouse Management gewährleistet. So ist eine aktuelle Bestandsübersicht, gegliedert nach Lagerzonen, jederzeit abrufbar. In zukünftigen Szenarien soll eine automatische Disposition die Beschaffungsprozesse ergänzen. Dafür sind ein automatisierter Bestellabruf bei Unterschreiten eines Materialmindestbestandes sowie eine anschließende automatische Transportgenerierung beim Lieferanten zu erstellen. 
Als weiterführendes Konzept ist ebenfalls die vollständige Abbildung der Unternehmenslandschaft und der Transportverbindungen durch die Software visTABLE vorgesehen. Derzeit ist die physische Struktur des OEM samt dessen innerbetrieblichen Transportsystemen in visTABLE modelliert.

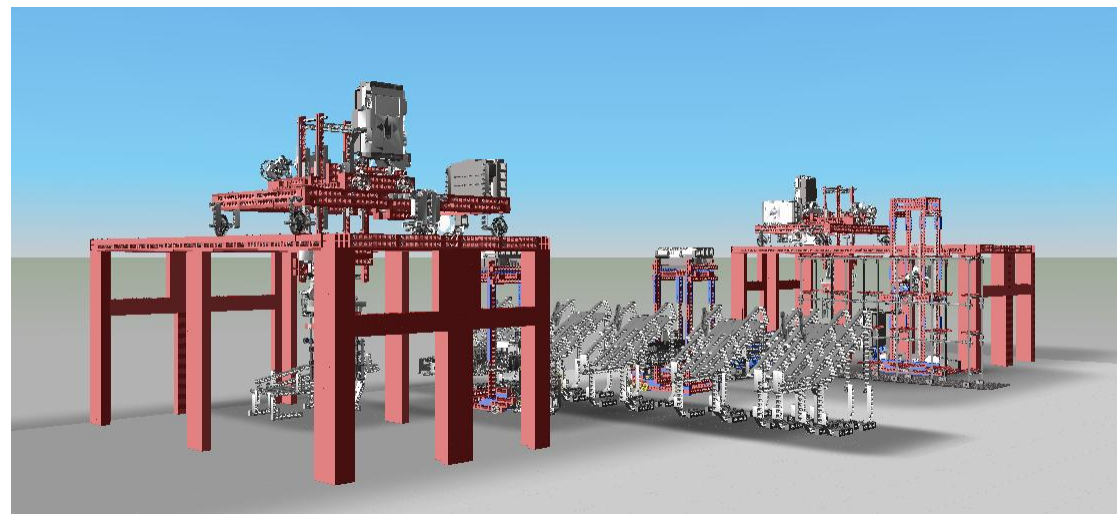

\section{Abbildung 4: Fabriklayout OEM in visTABLE}

Diese Struktur soll durch die Anbindung der externen Transportsysteme erweitert werden. Damit wird das Ziel verfolgt, den Wareneingangsprozess zu visualisieren und Analysen zum Prozessaufwand bei Bedarfsveränderungen vorzubereiten.

Die Verbindung dieser Technologien an den Schnittstellen zu SAP ERP ermöglicht eine virtuelle Planung von Fabriken sowie die Gestaltung von Logistikszenarien. Der Einsatz von visTABLE ermöglicht weitreichende Darstellungen von Geschäftsprozessen und deren physischen Schnittstellen zu geringen Kosten. Eine platz- und kostspielige physische Modellierung kann somit ersatzweise für interessierte Gymnasien oder Vertreter von Forschungspartnern angeboten werden.

Für die Kurse des Wintersemesters 2009/10 ist bereits eine Überarbeitung des Gesamtszenarios geplant und angestoßen. So soll die klassische Stuhlproduktion in der SC durch die modulbasierte Fertigung eines Kraftfahrzeuges ersetzt werden. Sowohl das Auftragsmanagement als auch die Bestandsführung sollen in weiten Teilen durch die Einführung einer Leitstandslösung zur Fertigungssteuerung in SAP SCM, mit dem Advanced Planning and Optimization (APO) bedient werden.

\section{Zusammenfassung}

Dieser Beitrag stellt die Einbindung eines weitgehend interaktiven Fallstudienkonzeptes mit einem ERP-System in der akademischen Lehre vor. Mit der Fallstudie wird das Lehrangebot der Fachgruppe Wirtschaftsinformatik angereichert. Weiterhin werden vielfältige Studien- und Lehrinhalte aller Studienmodule in kompakter 
Form verdichtet und praxis- sowie lebensnah aufbereitet. Der Kurs wird von Studierenden und Dozenten als nutzbringende Plattform zur Vertiefung studiengangsübergreifender Betrachtungen geschätzt. Er bereitet die Studierenden in ansprechender Weise auf die betrieblichen Anwendungssysteme, Schnittstellenproblematiken und betriebswirtschaftlichen Inhalte der Industrie vor. Darüber hinaus bietet der Kurs den studentischen Dozenten die Möglichkeit, individuelle Studienschwerpunkte im Umfeld einer ERP-Anwendung zu vertiefen. Nach internen Befragungen wird vor allem die Arbeitsorganisation in Teams zur Ausarbeitung und Abstimmung spezifischer Inhalte in den Kernfeldern von den Dozenten/Studierenden geschätzt. Für die praxisorientierte Vorbereitung der Studierenden auf Anforderungen im späteren Berufsleben stellt das einen bedeutenden Nutzen dar.

Durch die Verbindung der ERP/SCM-Komponenten mit dem SAP LEGO Labor konnten bisweilen interessante Verbindungen zu Industrie- und Forschungspartnern der Hochschule auf- bzw. ausgebaut werden. Das Labor dient ferner der Präsentation aktueller Forschungsarbeiten für Schüler im Rahmen der First Lego League. Somit bedient es die (vor-)akademische Ausbildung im Sinne der Zukunftssicherung der Hochschule.

\section{Literatur}

Daun C, Theling T und Loos P (2006) ERPeL - Blended Learning in der ERPLehre. Institut für Wirtschaftsinformatik, Heft 188, Saarbrücken

Eicker S, Kress S und Mense L (2007) ERP-Elearn eLearning für ERP-Systeme im Hochschulbereich am Beispiel von SAP R/3. In: Breitner MH, Bruns B und Lehner F (Hrsg.) Neue Trends im E-Learning - Aspekte der Betriebswirtschaftslehre und Informatik, Physika Verlag, Heidelberg

Grabot B, Bazet I und Mayère A (2008) ERP-Systems and organisational Change, A socio-technical insight. Springer online

Käschel J und Teich T(2008) Produktionswirtschaft, Band 3: Eine integrierte betriebswirtschaftliche Fallstudie mit SAP®. GUC, Chemnitz.

o.V. (2009) SAP-Ausbildung mit Lego

http://www.heise.de/newsticker/Hannover-Messe-SAP-Ausbildung-mit-Lego-/meldung/136598

Unger K, Militzer J and Teich T (2008) Visualizing Supply Chain Structures and

Processes to Evolve a Better Understanding for IT-related Operations in SAP ERP. In: Proceedings of CEISIE'08, Guangzhou (China) 